TITLE:

Structural similitude for the
geometric nonlinear buckling of
stiffened orthotropic shallow
spherical shells by energy approach

AUTHOR(S):

WANG, Jingchao; LI, Zhengliang; YU, Wei

CITATION:

WANG, Jingchao ... [et al]. Structural similitude for the geometric nonlinear buckling of stiffened orthotropic shallow spherical shells by energy approach. Japan-China Workshop on Analysis and Optimization of Large-scale Structures 2018: 10-10: A07.

ISSUE DATE:

2018-05-14

URL:

http://hdl.handle.net/2433/231240

RIGHT: 


\title{
Structural similitude for the geometric nonlinear buckling of stiffened orthotropic shallow spherical shells by energy approach
}

\author{
Jingchao WANG ${ }^{1^{*}}, \quad$ Zhengliang $\mathrm{LI}^{1,2}, \quad$ Wei YU $^{3}$ \\ ${ }^{1}$ School of Civil Engineering, Chongqing University, Chongqing 400045, China \\ (davidfwl@126.com) \\ ${ }^{2}$ Key Laboratory of New Technology for Construction of Cities in Mountain \\ Area(Chongqing University), Ministry of Education, Chongqing 400045, China \\ ${ }^{3}$ School of Civil Engineering, Guilin University of Technology, Qixing District, Guilin, \\ China
}

Keywords: structural similitude, stiffened orthotropic shallow spherical shells, the geometric nonlinear buckling, energy approach, partial similitude

\begin{abstract}
General similitude requirements and the scaling laws for nonlinear buckling of stiffened orthotropic shallow spherical shells are presented by applying similitude transformation to the total energy of the structural system. In the absence of the experimental data, structural similitude is completed by numerical experiments. The predicted values of the prototype, obtained by substituting the model results into the scaling laws, are compared with those values of the prototype. In practical engineering, it may be hard to fulfill the complete similarity requirements. Thus, several cases of partial similitude are mainly investigated, including models distorted in material properties of ribs, in material properties and in material properties and geometry. By using specific formulas of stiffness parameters and the displacement scale factor, distorted models can predict geometric nonlinear buckling behavior of the prototypes of the stiffened orthotropic shallow spherical shell under external pressure with good accuracy.
\end{abstract}

\section{References}

[1] W.T. Koiter, On the stability of elastic equilibrium, Thesis, Delft, English Translation, NASA TT-F10, 883 (1967) and AFFDL TR70-25 (1970)

[2] L.H. Donnell, C.C. Wan, Effect of imperfections on buckling of thin cylinders and columns under axial compression, (17) (1950). 